\title{
EXISTENCE OF HOMOCLINIC ORBITS FOR SECOND ORDER HAMILTONIAN SYSTEMS WITHOUT (AR) CONDITION
}

\author{
LI-LI WAN \\ (Communicated by C. L. Tang)
}

Abstract. The existence of homoclinic orbits is obtained for a class of the second order Hamiltonian systems $\ddot{u}(t)-L(t) u(t)+\nabla W(t, u(t))=0, \forall t \in \mathbb{R}$, by the mountain pass theorem, where $W(t, x)$ needs not to satisfy the global (AR) condition.

\section{Introduction and main result}

Let us consider the second order Hamiltonian systems

$$
\ddot{u}(t)-L(t) u(t)+\nabla W(t, u(t))=0, \quad \forall t \in \mathbb{R},
$$

where $L \in C\left(\mathbb{R}, \mathbb{R}^{N^{2}}\right)$ is a symmetric matrix valued function, $W \in C^{1}\left(\mathbb{R} \times \mathbb{R}^{N}, \mathbb{R}\right)$ and $\nabla W(t, x)=\frac{\partial W}{\partial x}(t, x)$. We say that a solution $u$ of problem (1) is homoclinic (to 0 ) if $u \in C^{2}\left(\mathbb{R}, \mathbb{R}^{N}\right), u(t) \rightarrow 0$ as $|t| \rightarrow \infty$. Homoclinic orbits of dynamical systems are important in the applications. From their existence and under certain conditions, one may infer the existence of chaos nearby or the bifurcation behavior of periodic orbits.

With the variational methods, the existence and multiplicity of homoclinic orbits of problem (1) have been obtained by many papers (see [1-4, 6-20]). But in most superquadratic cases, there is a so-called global (AR) condition on $W$, that is, there exists a constant $\mu>2$ such that

$$
0<\mu W(t, x) \leqslant(\nabla W(t, x), x), \forall t \in \mathbb{R}, \forall x \in \mathbb{R}^{N} \backslash\{0\},
$$

which is very important to guarantee the boundedness of the $(P S)_{c}$ sequence (see [1], [2], [4], [6], [8], [14], [15]).

Since the domain is unbounded, there is a lack of compactness of the Sobolev embedding. Many papers consider the periodic (autonomous, asymptotically periodic) problems (see [1], [2], [4], [7], [8], [14], [15]). Some papers treat the symmetric case (see [10], [11]). Recently the coercive case has been dealt with, that is,

$$
\text { the smallest eigenvalue of } L(t) \rightarrow+\infty \text { as }|t| \rightarrow \infty,
$$

(see [6], [12], [13], [15], [16], [19], [20]).

Without (2), we study the existence of homoclinic orbits of problem (1) without any periodic, symmetric or coercive assumption.

Mathematics subject classification (2000): 34C37, 37J45, 47J30, $58 \mathrm{E} 05$.

Keywords and phrases: homoclinic orbits, second order Hamiltonian systems, mountain pass theorem.

Supported by Youth Foundation of Southwest University of Science and Technology (No.09zx3127). 
THEOREM 1.1. Assume that $L \in C\left(\mathbb{R}, \mathbb{R}^{N^{2}}\right)$ and $W \in C^{1}\left(\mathbb{R} \times \mathbb{R}^{N}, \mathbb{R}\right)$ satisfy:

$\left(V_{1}\right) L(t)$ is uniformly positive definite;

$\left(V_{2}\right)$ there exist a bounded set $B \subset \mathbb{R}$ with int $(B) \neq \emptyset$ and $\mu>2, \theta>\frac{\mu}{\mu-2}$ such that:

(i) $0<\mu W(t, x) \leqslant(\nabla W(t, x), x), \forall t \in B, \forall x \in \mathbb{R}^{N} \backslash\{0\}$,

(ii) $0 \leqslant 2 W(t, x) \leqslant(\nabla W(t, x), x) \leqslant \frac{1}{\theta}(L(t) x, x), \forall t \notin B, \forall x \in \mathbb{R}^{N}$;

$\left(V_{3}\right)|\nabla W(t, x)| /|x| \rightarrow 0$ as $|x| \rightarrow 0$ uniformly in $t$.

Then problem (1) has at least one nontrivial homoclinic orbit.

REMARK 1.1 . Note that $W$ here needs not to satisfy (2) and $L$ needs not to satisfy the condition (3).

REMARK 1.2. Condition $\left(V_{2}\right)$ is inspired by Condition $(g 3)$ in [5].

EXAMPLE 1.1. There exist the functions $W(t, x)$ and $L(t)$ which satisfy the conditions of Theorem 1.1 but do not satisfy (2) and (3) respectively. For example, let $L(t) \equiv 12, \mu=3, \theta=4$, and

$$
f(x)= \begin{cases}|x|^{3}, & |t| \leqslant 1, x \in \mathbb{R}^{N} \\ |x|^{3}, & |t|>1,|x| \leqslant 1 \\ |x|^{2}, & |t|>1,|x|>2\end{cases}
$$

\section{Proof of Theorem 1.1}

Define

$$
H=\left\{u \in H^{1}\left(\mathbb{R}, \mathbb{R}^{N}\right): \int_{\mathbb{R}}\left(|\dot{u}|^{2}+(L(t) u, u)\right) d t<+\infty\right\} .
$$

Then $H$ is a Hilbert space equipped with the following norm:

$$
\|u\|=\left(\int_{\mathbb{R}}\left(|\dot{u}|^{2}+(L(t) u, u)\right) d t\right)^{\frac{1}{2}} .
$$

Obviously, by $\left(V_{1}\right)$, the space $H$ is continuously embedded in $H^{1}\left(\mathbb{R}, \mathbb{R}^{N}\right)$ and so continuously embedded in $L^{p}\left(\mathbb{R}, \mathbb{R}^{N}\right)$ for $p \in[2, \infty]$. Thus we have

$$
\|u\|_{L^{p}} \leqslant \gamma_{p}\|u\| \text { for } p \in[2, \infty],
$$

where $\gamma_{p}>0$. Set

$$
f(u)=\frac{1}{2} \int_{\mathbb{R}}\left(|\dot{u}|^{2}+(L(t) u, u)\right) d t-\int_{\mathbb{R}} W(t, u) d t=\frac{1}{2}\|u\|^{2}-\int_{\mathbb{R}} W(t, u) d t .
$$

It is easy to see that $f \in C^{1}\left(H, \mathbb{R}^{N}\right)$ and the critical point of $f$ in $H$ is the homoclinic orbit of problem (1). In the following, we always denote by $C_{i}(i \in N)$ any suitable 
positive constant.

Proof of Theorem 1.1 We divide our proof into three steps.

The first step. There are $\rho>0, \delta>0$ such that

$$
\left.f\right|_{S} \geqslant \delta
$$

where $S=\{u \in H \mid\|u\|=\rho\}$. In fact, it follows from $\left(V_{3}\right)$ that, for any $\varepsilon>0$, there exists $\rho^{\prime}=\rho^{\prime}(\varepsilon)>0$ such that

$$
|\nabla W(t, x)| \leqslant 2 \varepsilon|x|, \quad \forall t \in \mathbb{R}, \forall|x| \leqslant \rho^{\prime},
$$

which implies that

$$
|W(t, x)| \leqslant \varepsilon|x|^{2}, \quad \forall t \in \mathbb{R}, \forall|x| \leqslant \rho^{\prime} .
$$

Then choose

$$
\varepsilon=\frac{1}{4 \gamma_{2}^{2}}>0, \rho=\frac{\rho^{\prime}}{\gamma_{\infty}}>0 \text { and } \delta=\frac{\rho^{2}}{4}
$$

and we have

$$
\begin{aligned}
f(u) & =\frac{1}{2}\|u\|^{2}-\int_{\mathbb{R}} W(t, u) d t \\
& \geqslant \frac{1}{2}\|u\|^{2}-\varepsilon \int_{\mathbb{R}}|u|^{2} d t \\
& \geqslant \frac{1}{2}\|u\|^{2}-\varepsilon \gamma_{2}^{2}\|u\|^{2}=\frac{1}{4}\|u\|^{2}=\delta,
\end{aligned}
$$

for all $u \in S$.

The second step. There exists $u_{0} \in H$ such that $\left\|u_{0}\right\|>\rho$ and $f\left(u_{0}\right)<0$. In fact, by $\left(V_{2}\right)$ (i) we have

$$
W(t, x) \geqslant C_{1}|x|^{\mu}, \forall t \in B, \forall|x| \geqslant 1 .
$$

Now choose $\tilde{u} \in H \backslash\{0\}$ with its support contained in $B$, then using $\left(V_{2}\right)$ (i), Fatou Lemma and (4) one has

$$
\begin{aligned}
\limsup _{s \rightarrow+\infty} \frac{f(s \tilde{u})}{s^{2}} & =\frac{1}{2}\|\tilde{u}\|^{2}-\liminf _{s \rightarrow+\infty} \int_{\mathbb{R}} \frac{W(t, s \tilde{u})}{s^{2}} d t \\
& =\frac{1}{2}\|\tilde{u}\|^{2}-\liminf _{s \rightarrow+\infty} \int_{B \backslash\{t:|\tilde{u}(t)|=0\}} \frac{W(t, s \tilde{u}) \tilde{u}^{2}}{s^{2} \tilde{u}^{2}} d t \\
& \leqslant \frac{1}{2}\|\tilde{u}\|^{2}-\int_{B \backslash\{t:|\tilde{u}(t)|=0\}} \liminf _{s \rightarrow+\infty} \frac{W(t, s \tilde{u}) \tilde{u}^{2}}{s^{2} \tilde{u}^{2}} d t<0,
\end{aligned}
$$

so $u_{0}$ exists. 
The two steps above imply that $f$ has the mountain pass geometry and then there exist a positive constant $c$ and a $(P S)_{c}$ sequence $\left(u_{n}\right) \subset H$ at the level $c$, that is,

$$
f\left(u_{n}\right) \rightarrow c \text { and }\left\|f^{\prime}\left(u_{n}\right)\right\| \rightarrow 0 \text { as } n \rightarrow \infty .
$$

The third step. $f$ satisfies the $(P S)_{c}$ condition. First we show that $\left(u_{n}\right)$ is bounded. By (5) we have

$$
o\left(\left\|u_{n}\right\|\right)=\left\langle f^{\prime}\left(u_{n}\right), u_{n}\right\rangle=\int_{\mathbb{R}}\left(\left|\dot{u}_{n}\right|^{2}+\left(L(t) u_{n}, u_{n}\right)\right) d t-\int_{\mathbb{R}}\left(\nabla W\left(t, u_{n}\right), u_{n}\right) d t,
$$

which combined with $\left(V_{2}\right)$ implies that

$$
\begin{aligned}
\int_{\mathbb{R}}\left(|\dot{u}|^{2}+(L(t) u, u)\right) d t & =\int_{\mathbb{R}}\left(\nabla W\left(t, u_{n}\right), u_{n}\right) d t+o\left(\left\|u_{n}\right\|\right) \\
& \geqslant \int_{B}\left(\nabla W\left(t, u_{n}\right), u_{n}\right) d t+o\left(\left\|u_{n}\right\|\right) \\
& \geqslant \mu \int_{B} W\left(t, u_{n}\right) d t+o\left(\left\|u_{n}\right\|\right) .
\end{aligned}
$$

It follows from (5), $\left(V_{2}\right)$ (ii) and (6) that

$$
\begin{aligned}
\frac{1}{2} \int_{\mathbb{R}}\left(\left|\dot{u}_{n}\right|^{2}+\left(L(t) u_{n}, u_{n}\right)\right) d t \leqslant & \int_{\mathbb{R}} W\left(t, u_{n}\right) d t+C_{2} \\
& =\int_{B} W\left(t, u_{n}\right) d t+\int_{\mathbb{R} \backslash B} W\left(t, u_{n}\right) d t+C_{2} \\
& \leqslant \int_{B} W\left(t, u_{n}\right) d t+\frac{1}{2 \theta} \int_{\mathbb{R} \backslash B}\left(L(t) u_{n}, u_{n}\right) d t+C_{2} \\
\leqslant & \frac{1}{\mu} \int_{B}\left(\left|\dot{u}_{n}\right|^{2}+\left(L(t) u_{n}, u_{n}\right)\right) d t+o\left(\left\|u_{n}\right\|\right) \\
& +\frac{1}{2 \theta} \int_{\mathbb{R} \backslash B}\left(L(t) u_{n}, u_{n}\right) d t+C_{2} .
\end{aligned}
$$

Since by $\left(V_{2}\right)$ (ii) one has $\frac{\mu}{2}-1>\frac{\mu}{2 \theta}$, by (7) we get

$$
\left(\frac{\mu}{2}-1-\frac{\mu}{2 \theta}\right)\left\|u_{n}\right\|^{2} \leqslant o\left(\left\|u_{n}\right\|\right)+C_{2},
$$

that is,

$$
\left\|u_{n}\right\| \leqslant C_{3} .
$$

Now passing to a subsequence if necessary, we may assume that $u_{n} \rightarrow u$ as $n \rightarrow \infty$ for some $u \in H$. Second, we show that the convergence is strong. It is easy to see that we need only to prove that for any $\varepsilon>0$, there exists an $r=r(\varepsilon)>0$ such that

$$
\limsup _{n \rightarrow \infty} \int_{\mathbb{R} \backslash B_{r}}\left(\left|\dot{u}_{n}\right|^{2}+\left(L(t) u_{n}, u_{n}\right)\right) d t<\varepsilon,
$$


where $B_{r}$ is the open ball with center 0 and radius $r$. Since $B$ is bounded, we may choose $r$ large enough that $B \subset B_{\frac{r}{2}}$. Now let $\eta_{r}$ be a cut-off function satisfying that:

$$
\eta_{r}=0 \text { on } B_{\frac{r}{2}}, \eta_{r}=1 \text { on } \mathbb{R} \backslash B_{r}, 0 \leqslant \eta_{r} \leqslant 1 \text { and }\left|\dot{\eta}_{r}\right| \leqslant \frac{C_{4}}{r} .
$$

It follows from (5) and (8) that

$$
\left\langle f^{\prime}\left(u_{n}\right), \eta_{r} u_{n}\right\rangle=o(1)
$$

that is,

$$
\begin{aligned}
\int_{\mathbb{R}}\left(\left(\left|\dot{u}_{n}\right|^{2}+\left(L(t) u_{n}, u_{n}\right)\right)\right) & \eta_{r} d t+\int_{\mathbb{R}}\left(\dot{u}_{n}, u_{n}\right) \dot{\eta}_{r} d t \\
= & \int_{\mathbb{R}}\left(\nabla W\left(t, u_{n}\right), u_{n} \eta_{r}\right) d t+o(1),
\end{aligned}
$$

which combined with $\left(V_{2}\right)$ (ii) implies that

$$
\begin{aligned}
\int_{\mathbb{R}}\left(\left(\left|\dot{u}_{n}\right|^{2}+\left(L(t) u_{n}, u_{n}\right)\right)\right) \eta_{r} d t \\
\quad=-\int_{\mathbb{R}} \dot{u}_{n} u_{n} \dot{\eta}_{r} d t+\int_{\mathbb{R}}\left(\nabla W\left(t, u_{n}\right), u_{n} \eta_{r}\right) d t+o(1) \\
\quad \leqslant-\int_{\mathbb{R}} \dot{u}_{n} u_{n} \dot{\eta}_{r} d t+\frac{1}{\theta} \int_{\mathbb{R}}\left(L(t) u_{n}, u_{n}\right) \eta_{r} d t+o(1) .
\end{aligned}
$$

Now by (10), Hölder inequality and (8) one can see that

$$
\begin{aligned}
\left(1-\frac{1}{\theta}\right) & \int_{\mathbb{R} \backslash B_{r}}\left(\left|\dot{u}_{n}\right|^{2}+\left(L(t) u_{n}, u_{n}\right)\right) d t \\
& \leqslant \frac{C_{4}}{r}\left\|u_{n}\right\|_{L^{2}}\left\|\dot{u}_{n}\right\|_{L^{2}}+o(1) \\
& \leqslant \frac{\gamma_{2} C_{3}^{2} C_{4}}{r}+o(1),
\end{aligned}
$$

which implies (9) immediately. Therefore, $u$ is the nontrivial homoclinic orbit of problem (1). The proof is complete.

\section{REFERENCES}

[1] C. O. Alves, P. C. Carriao and O. H. Miyagaki, Existence of homoclinic orbits for asymptotically periodic systems involving Duffing-like equation, Appl. Math. Lett., 16, 5 (2003), 639-642.

[2] V. Coti-Zelati And P. H. Rabinowitz, Homoclinic orbits for second order Hamiltonian systems possessing superquadratic potentials, J. Amer. Math. Soc., 4, 4 (1991), 693-727.

[3] V. COTI-Zelati, I. EKeland And E. SeRE, A variational approach to homoclinic orbits in Hamiltonian systems, Math. Ann., 288, 1 (1990), 133-160.

[4] P. C. CARRi Ão AND O. H. MiYAGAKI, Existence of homoclinic solutions for a class of timedependent Hamiltonian systems, J. Math. Anal. Appl., 230 (1999), 157-172. 
[5] M. Del Pino, Patricio L. Felmer, Local mountain passes for semilinear elliptic problems in unbounded domains, Calc. Var. Partial Differential Equations, 4, 2 (1996), 121-137.

[6] Y. H. DiNG, Existence and multiplicity results for homoclinic solutions to a class of Hamiltonian systems, Nonlinear Anal., 25 (1995), 1095-1113.

[7] P. L. Felmer And E. A. De B. E Silva, Homoclinic and periodic orbits for Hamiltonian systems, Ann. Scuola Norm. Sup. Pisa Cl. Sci., 26 (1998), 285-301.

[8] M. IZYDOREK AND J. JANCZEWSKA, Homoclinic solutions for a class of the second order Hamiltonian systems, J. Differential Equations, 219, 2 (2005), 375-389.

[9] M. IZYDOREK AND J. JANCZEWSKA, Homoclinic solutions for nonautonomous second order Hamiltonian systems with a coercive potential, J. Math. Anal. Appl., 335 (2007), 1119-1127.

[10] P. Korman, A. C. Lazer, Homoclinic orbits for a class of symmetric Hamiltonian systems, Electron. J. Differential Equations, 1994 (1994), 1-10.

[11] Y. LV, C. L. TANG, Existence of even homoclinic orbits for second-order Hamiltonian systems, Nonlinear Anal., 67, 7 (2007), 2189-2198.

[12] W. Omana AND M. Willem, Homoclinic orbits for a class of Hamiltonian systems, Differential Integral Equations, 5, 5 (1992), 1115-1120.

[13] Z. Q. OU AND C. L. TANG, Existence of homoclinic solution for the second order Hamiltonian systems, J. Math. Anal. Appl., 291, 1 (2004), 203-213.

[14] P. H. RABINOwITZ, Homoclinic orbits for a class of Hamiltonian systems, Proc. Roy. Soc. Edinburgh, Sect. A, 114 (1990), 33-38.

[15] P. H. RABINOWITZ AND K. TANAKA, Some results on connecting orbits for a class of Hamiltonian systems, Math. Z., 206 (1990), 473-499.

[16] A. Salvatore, Multiple homoclinic orbits for a class of second order perturbed Hamiltonian systems, Dynamical systems and differential equations (Wilmington, NC, 2002), Discrete Contin. Dyn. Syst., 2003, suppl., 778-787.

[17] S. P. WU AND J. Q. LIU, Homoclinic orbits for second order Hamiltonian system with quadratic growth, Appl. Math. J. Chinese Univ. Ser. B, 10 (1995), 399-410.

[18] S. P. WU AND H. T. YANG, A note on homoclinic orbits for second order Hamiltonian system, Appl. Math. J. Chinese Univ. Ser. B, 13 (1998), 251-262.

[19] J. YANG AND F. B. ZHAO, Infinitely many homoclinic orbits for the second-order Hamiltonian systems with super-quadratic potentials, Nonlinear Analysis: Real World Applications, 10, 3 (2009), $1417-1423$.

[20] W. M. ZoU AND S. J. LI, Infinitely many homoclinic orbits for the second-order hamiltonian systems, Appl. Math. Lett., 16, 8 (2003), 1283-1287.

(Received June 7, 2009)

Li-Li Wan

School of Science

Southwest University of Science and Technology

Mianyang Sichuan 621010

China

e-mail: valley_527@163.com 